OPEN

SUBJECT AREAS:

MAGNETIC PROPERTIES

AND MATERIALS

MATERIALS SCIENCE

Received

27 August 2013

Accepted

15 October 2013

Published

4 November 2013

Correspondence and requests for materials should be addressed to R.S. (sooryakumar.1@ osu.edu)

\section{Patterned time-orbiting potentials for the confinement and assembly of magnetic dipoles}

\author{
A. Chen \& R. Sooryakumar
}

Department of Physics, The Ohio State University, Columbus, Ohio 43210, USA.

We present an all-magnetic scheme for the assembly and study of magnetic dipoles within designed confinement profiles that are activated on micro-patterned permalloy films through a precessing magnetic field. Independent control over the confinement and dipolar interactions is achieved by tuning the strength and orientation of the revolving field. The technique is demonstrated with superparamagnetic microspheres field-driven to assemble into closely packed lattice sheets, quasi-1D and other planar structures expandable into dipolar arrays that mirror the patterned surface motifs.

The he confinement and manipulation of charge carriers within intricate low-dimensional structures through designed electric potentials have led to revolutionary advances in semiconductor devices ${ }^{1}$. In contrast, the magnetic analog of creating designable energy landscapes for confining magnetic dipoles with concomitant tunability of the relevant forces solely through magnetic fields has however remained elusive. Such field-based approaches could offer powerful means to probe mesoscopic magnetic systems characterized by length scales ranging from tens of nanometers to micrometers, forces extending from femto- to nano-newtons and times scales above a microsecond.

Schemes to confine interacting magnetic dipoles of micrometer to centimeter scale have been proposed as early as in $1878^{2}$. However, existing approaches ${ }^{2-7}$ confront challenges such as hard-wall confinements that prohibit regulation of the size of the dipole cluster, restriction of magnetic dipolar interaction to either repulsive or attractive, and lack of control on introducing and removing a specific number of dipoles (smaller than millimeter size) within the interacting landscape. Magnetic traps have also been developed for the confinement of dipolar atoms ${ }^{8-13}$; however, the current-carrying coils utilized offer limited configurations on the spatial design and size of the trap.

Here we present a straightforward mechanism that overcomes these challenges by activating a time-orbiting potential on a micro-patterned permalloy thin film with a precessing magnetic field. Independent tunability of the soft-confinement force and dipolar interactions (reversibly between attraction and repulsion) as well as in-situ control on the number of confined dipoles are obtained solely through tuning the magnetic field. Moreover, the lithographically defined confinement scheme allows complex designer landscapes of desired length scales to be readily fabricated. Demonstrated by the remote, field-driven assembly of dipoles consisting of fluid-borne microspheres, this all-magnetic scheme serves as a prototype to stabilize various low-dimensional spatial constructs realizable over wide length scales. These features enable fundamental studies such as artificial atoms ${ }^{14-18}$, nucleation ${ }^{7,19-24}$, jamming ${ }^{25-27}$ and frustration ${ }^{28-30}$ as well as applications in biomedicine ${ }^{31-39}$, material assembly ${ }^{40-45}$, photonics $^{5,46-49}$, magnetic logic ${ }^{50-52}$, chip based devices ${ }^{53-57}$ and atom traps ${ }^{8-13}$.

\section{Results}

The architectures of the confining potential for magnetic dipoles are defined by permalloy $\left(\mathrm{Ni}_{0.8} \mathrm{Fe}_{0.2}\right)$ thin-film patterns imprinted on a silicon substrate through photo or electronbeam lithography(see Methods section). Figure 1(a) schematically illustrates a random distribution of superparamagnetic microspheres $(8 \mu \mathrm{m}$ in diameter, UMC4F/9560, Bangs Laboratories) in solution on a permalloy disk pattern $(210 \mu \mathrm{m}$ in diameter, $40 \mathrm{~nm}$ thick) that have, in the absence of magnetic fields, been drawn to the surface by gravity. An externally applied field $\mathrm{H}_{\mathrm{ext}}=\left(H_{x}, H_{y}, H_{z}\right)$ transforms the microspheres into dipoles while the permalloy disk becomes uniformly magnetized along the $\left(H_{x}, H_{y}, 0\right)$ direction. The dipoles interact with each other as well as fields emanating from the disk $\mathrm{s}^{58-60}$, arranging themselves into configurations that minimize the total energy $U$, that has primary 

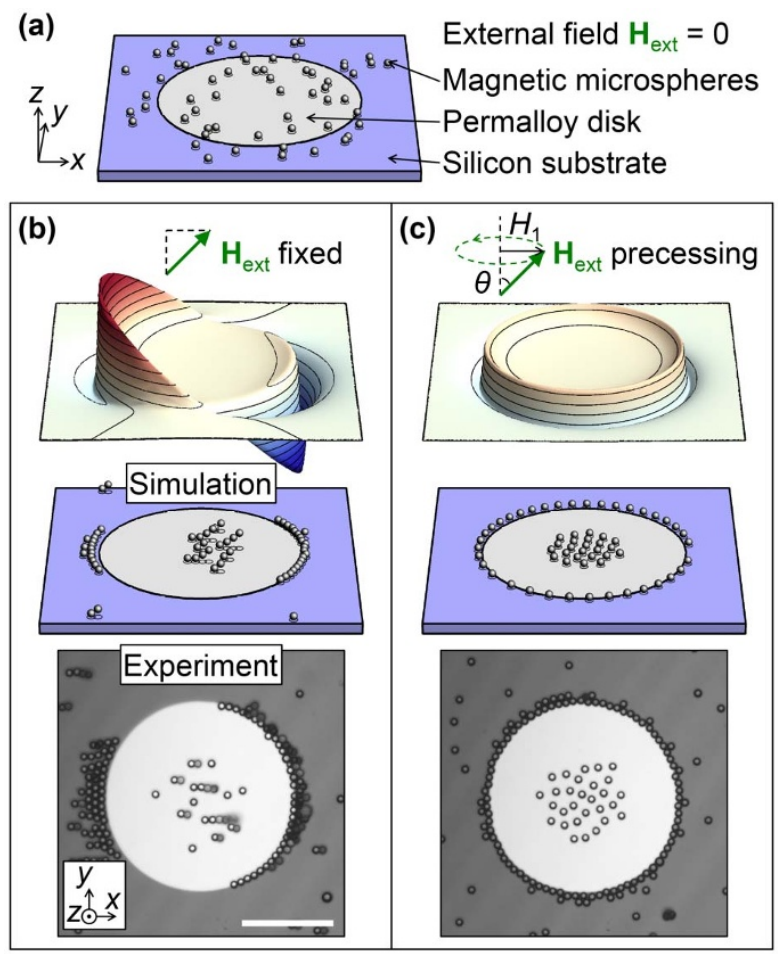

Figure 1 Formation of a time-orbiting potential on a permalloy disk. (a) Illustration of magnetic microspheres randomly distributed atop a permalloy disk within fluid environment on a silicon substrate in the absence of external magnetic fields. (b) In the presence of $\mathbf{H}_{\text {ext }}=(60,0,60)$ $\left(\times 10^{3} / 4 \pi\right) \mathrm{A} / \mathrm{m}$, shown from top to bottom are the calculated energy landscape $\left(U_{\text {disk }}\right)$, simulation, and experimental snapshot of the system in equilibrium. Contour interval on the energy landscape is $2000 k_{\mathrm{B}} T$, where $k_{\mathrm{B}}$ is Boltzmann's constant and $T=300 \mathrm{~K}$ the temperature. Projections of the microspheres on the surface are drawn in circles in the simulation to show levitation of microsphere chains along $\mathbf{H}_{\text {ext }}$. Scale bar in the snapshot is $100 \mu \mathrm{m}$. (c) Same as in (b) but with $\mathbf{H}_{\text {ext }}$ precessing about the $z$-axis at $20 \mathrm{~Hz}$ with in-plane component $H_{1}=60\left(\times 10^{3} / 4 \pi\right) \mathrm{A} / \mathrm{m}$ and angle of precession $\theta=45^{\circ}$, leading to a time-averaged energy landscape, $\overline{U_{\text {disk }}}$.

contributions from the dipole-disk $\left(U_{\text {disk }}\right)$ and dipole-dipole $\left(U_{\text {dip }}\right)$ interaction energies (see Supplementary Information). As shown in Fig. 1(b), in the presence of a fixed, oblique external field of $\mathrm{H}_{\text {ext }}=$ $(60,0,60)\left(\times 10^{3} / 4 \pi \mathrm{A} / \mathrm{m}[=(60,0,60)\right.$ Oe $\left.]\right)$ oriented between the $+x$ and $+z$ directions, each dipole on the plane above the pattern experiences an asymmetric energy landscape, $U_{\text {disk, }}$, where a strong energy barrier and trench exist at the disk edges facing the $-x$ and $+x$ directions respectively. The typical force experienced by a microsphere (lying within a circular area of radius $80 \%$ that of the disk) is less than $1 \mathrm{pN}$, which corresponds to a time scale of greater than $1.5 \mathrm{~s}$ for the dipole to move a distance equal to its own diameter. Therefore, precessing $\mathrm{H}_{\text {ext }}$ about the $z$-axis at $20 \mathrm{~Hz}$ (time period $0.05 \mathrm{~s}$ ) results in the microsphere motion being insensitive to the fast varying component of the energy profile. Instead, the dipoles on the patterned disk respond to a time-averaged energy landscape, $\overline{U_{\text {disk }}}$, in the form of a bowl-shaped confinement profile surrounded by a circular trench as shown in Fig. 1(c). The precessing field also renders rotational symmetry to the time-averaged dipole-dipole interaction $\left(\overline{U_{\text {dip }}}\right)$, facilitating formation of an ordered planar cluster rather than aggregated chains within the confining landscape. As discussed below, the time-averaged forms of these interactions are utilized for the confinement and assembly of the microsphere (dipole) cluster.

We first obtain a quantitative understanding on the interplay between the two primary forces - the confinement force, (a) Confinement force $(\mathrm{pN})$ (b) Dipolar force $(\mathrm{pN})$
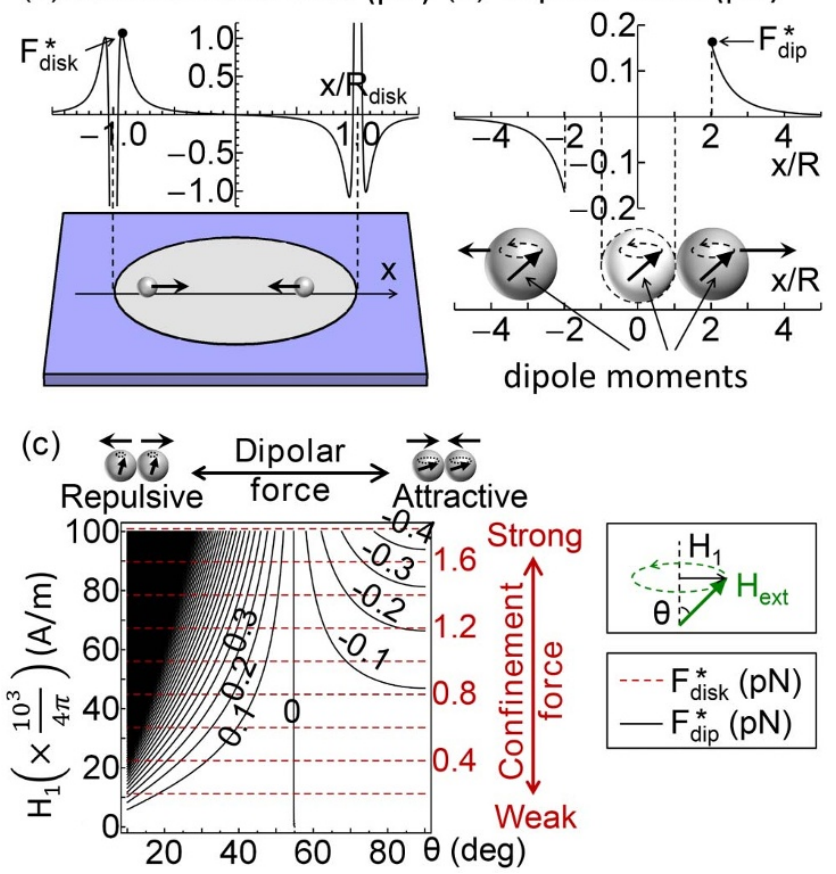

Figure $2 \mid$ Quantification of the two primary forces in the system: timeaveraged confinement force $\left(\overline{\mathbf{F}_{\text {disk }}}\right)$ and dipolar force $\left(\overline{\mathbf{F}_{\text {dip }}}\right)$. In the presence of a precessing $\mathbf{H}_{\text {ext }}$ as in Fig. 1(c), the in-plane ( $x$ ) component of (a) $\overline{\mathbf{F}_{\text {disk }}}$ and (b) $\overline{\mathbf{F}_{\text {dip }}}$ (exerted by another microsphere located at $x=0$ ) are plotted as a function of $x$ (in units of the disk radius $R_{\text {disk }}$ and microsphere radius $R) . F_{\text {disk }}^{*}$ and $F_{\text {dip }}^{*}$ are defined as the maximum in-plane confinement and dipolar forces respectively. (c) Contours of constant $F_{\text {disk }}^{*}$ and $F_{\text {dip }}^{*}$ are plotted in the parameter space spanned by $H_{1}$ and $\theta$.

$\overline{\mathbf{F}_{\text {disk }}}=-\nabla \overline{U_{\text {disk }}}$, that arise from the dipole-disk interaction and the dipolar force, $\overline{\mathbf{F}_{\text {dip }}}=-\nabla \overline{U_{\text {dip }}}$. In the presence of a precessing $\mathbf{H}_{\text {ext }}$ oriented at, for example, $\theta=45^{\circ}$ from the $z$-axis with an in-plane component $H_{1}=60\left(\times 10^{3} / 4 \pi\right) \mathrm{A} / \mathrm{m}$, the confinement force experienced by a microsphere atop the disk is $<1 \mathrm{pN}$ directed towards the center as shown in Fig. 2(a). An interesting property is the sole dependence of the confinement force on $H_{1}$, since $\overline{\mathbf{F}_{\text {disk }}} \propto H_{1}$. Figure 2(b) shows that under the same precessing field, the corresponding dipolar force between two microspheres on the surface is $\sim 0.2 \mathrm{pN}$ (repulsive) and weakens with their separation $(x)$ as $1 / x^{4}$. Moreover, since $\overline{\mathbf{F}_{\text {dip }}} \propto H_{1}^{2}\left(2 \cot ^{2} \theta-1\right)$, the dipolar force can be tuned from being repulsive $\left(\theta<\theta_{\mathcal{c}}\right)$ to attractive $\left(\theta>\theta_{\mathrm{c}}\right)$, crossing over at $\theta=\theta_{c}=\cot ^{-1}(1 / \sqrt{2}) \approx 54.7^{\circ}$. Separate and independent control on the confinement and dipolar forces can thus be obtained, as illustrated in Fig. 2(c), by tuning $H_{1}$ and $\theta$ along contours of constant $H_{1}$ or constant $H_{1}^{2}\left(2 \cot ^{2} \theta-1\right)$. Derivations for the above, including the expressions:

$$
\begin{aligned}
\overline{U_{\text {disk }}}(\mathbf{r})= & -\frac{\mu_{0} H_{1}}{8 \pi} \chi V M_{\text {disk }} d \\
& \frac{1}{\rho}\left[\left(2-m-4 \frac{R_{\text {disk }}^{2}}{\rho^{2}}\right) \Pi(m, m)-2 K(m)\right],
\end{aligned}
$$

and

$$
\overline{U_{\mathrm{dip}}}(\mathbf{r})=-\frac{\mu_{0}}{8 \pi} \chi^{2} V^{2} H_{\mathrm{ext}}^{2}\left(3 \cos ^{2} \theta-1\right) \sum_{\substack{i \\ \mathbf{r}_{i} \neq \mathbf{r}}} \frac{3 \cos ^{2} \alpha_{i}-1}{\left|\mathbf{r}-\mathbf{r}_{i}\right|^{3}},
$$

are available as Supplementary Information. 
The ability to tune the dipolar interaction (with $\theta$ ) while the softconfinement force is fixed (with $H_{1}$ ) allows the confined dipoles to assemble into various structures. An important parameter characterizing the dipole structure is the average center-to-center microsphere separation, $\langle\Delta r\rangle$, determined by Delaunay triangulation ${ }^{61}$ which serves as a measure of the cluster size. As shown in the plot of Fig. $3,\langle\Delta r\rangle$ is varied over a factor of two from $4 R$ to $2 R$ (in contact) as $\theta$ is increased from $35^{\circ}$ to $70^{\circ}$. In the repulsive regime of dipolar interaction $\left(\theta<\theta_{\mathrm{c}}\right)$, the dipoles form an expanded cluster within a center-attracting potential (insets A, B, C, F and G of Fig. 3), resembling the two-dimensional version of J. J. Thomson's atomic “plum pudding model" 62 and lending itself as an unstudied form of artificial atom $^{14-18}$. In the attractive regime $\left(\theta>\theta_{c}\right)$, the dipoles form a sheet of closely packed hexagonal lattice centered on the disk (insets D, E, I and J of Fig. 3). Due to the initial dipole configuration, occasional jamming - inability to form a single lattice - could occur (inset I and F2 of Fig. 3) that is related to gelations and glass transitions ${ }^{25-27}$ in bulk systems. The transition from a $2 \mathrm{D}$ plum pudding to an ordered lattice occurs at $\theta=\theta_{\mathrm{c}} \approx 54.7^{\circ}$ as borne out in the simulation where the lowest order dipolar interaction vanishes. A small deviation of the observed transitioning angle to $\theta_{\mathrm{c}} \sim 60^{\circ}$ in the experiment could arise from the weakly repulsive hydrodynamic forces due to rotational motion of the microspheres in a precessing field. The existence of multiple forms of competing interactions could cause frustra$\operatorname{tion}^{28-30}$, leading to formation of exotic structures such as the loosely

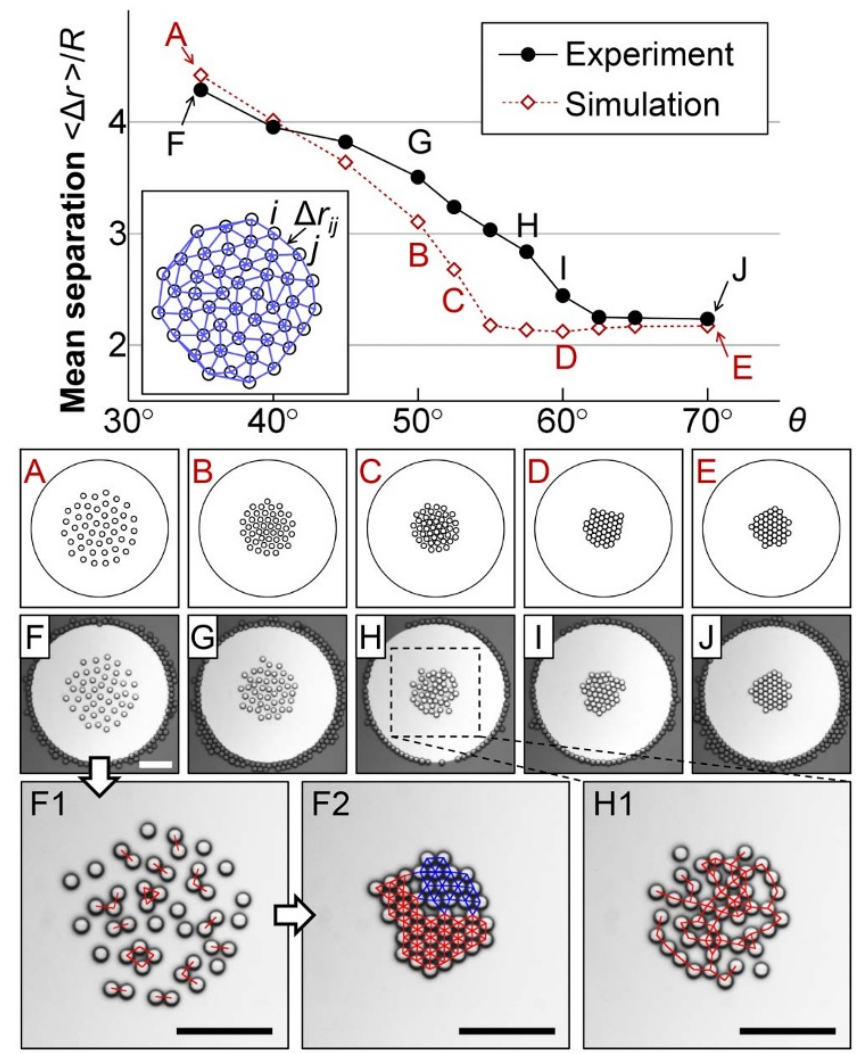

Figure $3 \mid$ Tuning the structure of a 45-microsphere cluster confined in the time-orbiting potential. The mean separation between microspheres, $\langle\Delta r\rangle$, determined by the bonding lengths given by Delaunay triangulation (lower left inset in the plot) is plotted as a function of $\theta$ while $H_{1}=60$ $\left(\times 10^{3} / 4 \pi\right) \mathrm{A} / \mathrm{m}$. Snapshots of the equilibrium microsphere configurations at corresponding values of $\theta$ are shown below for both simulation (insets $\mathrm{A}-\mathrm{E}$ ) and experiment (insets F-J). Insets F1 and F2 are snapshots taken at $12.6 \mathrm{~s}$ and $50.0 \mathrm{~s}$ after the instant switching of $\theta$ from $35^{\circ}$ to $90^{\circ}$, while inset $\mathrm{H} 1$ is an enlargement from inset $\mathrm{H}$. Lines are drawn between microspheres with less than $10 \mu \mathrm{m}$ center-to-center separation $(\sim 1.25$ times the diameter) in insets F1, F2 and H1. Scale bars are $50 \mu \mathrm{m}$. connected chains observed at $\theta=57.5^{\circ}$ (insets $\mathrm{H}$ and $\mathrm{H} 1$ of Fig. 3). In this case, competition sets in between the shorter-ranged dipolar attraction and longer-ranged hydrodynamic repulsion that scale with inter-particle distance $(x)$ as $1 / x^{4}$ and $1 / x^{3}$ respectively ${ }^{6}$. The soft-confining force acts as a catalyst in assisting the dipoles to overcome the energy barrier between them, thereby facilitating chain connections. In addition to equilibrium structures, transient effects are observed by abruptly changing the dipolar interaction (within $1 \mu \mathrm{s})$. For example, reorienting the precessing $\mathbf{H}_{\text {ext }}$ from the repulsive to attractive regime $\left(\theta=35^{\circ} \rightarrow 90^{\circ}\right)$ leads to nucleation of the cluster $^{7,19-24}-$ a first order phase transition that is often difficult to observe at the molecular scale and to reversibly produce in the experiments - as shown in inset F1 of Fig. 3. Reversible control on the cluster structure is demonstrated in Videos S1 and S2 of Supplementary Information, where $\theta$ is instantly switched from $90^{\circ}$ to $35^{\circ}$ (Video S1) and from $35^{\circ}$ to $90^{\circ}$ (Video S2) while $H_{1}$ is held fixed at $60\left(\times 10^{3} / 4 \pi\right) \mathrm{A} / \mathrm{m}$.

Crucial to the study of cluster structure is the control on the number of objects. However, such control for magnetic dipoles has only been achieved through direct physical placement into and out of the confinement for millimeter- and larger sized objects ${ }^{2,5,6}$ or through the random compartmentalization for much smaller objects $^{4,7}$. In contrast, by application of a sequence of fixed magnetic fields which disrupt the time-orbiting potential, or a rotating magnetic field (e.g. about the $x$ - or $y$-axis) which "rolls" the microspheres along targeted direction, a desired number of dipoles can be loaded into or unloaded from the confinement zone in the presented scheme. Examples of loading dipoles from 50 to 60, 70, 80, 90 and 100 followed by unloading are demonstrated in Videos S3 and S4 of Supplementary Information.

\section{Discussion}

The time-orbiting bowl-shaped profile can be readily extended to other shaped confining potentials with surrounding barriers and trenches defined by permalloy patterns. Shown in Fig. 4(a-d) are examples of triangle, square, long rectangle and octagonal ring geometries that maintain the same independent control on the confining and dipolar forces through $\theta$ and $H_{1}$. These energy landscapes unveil
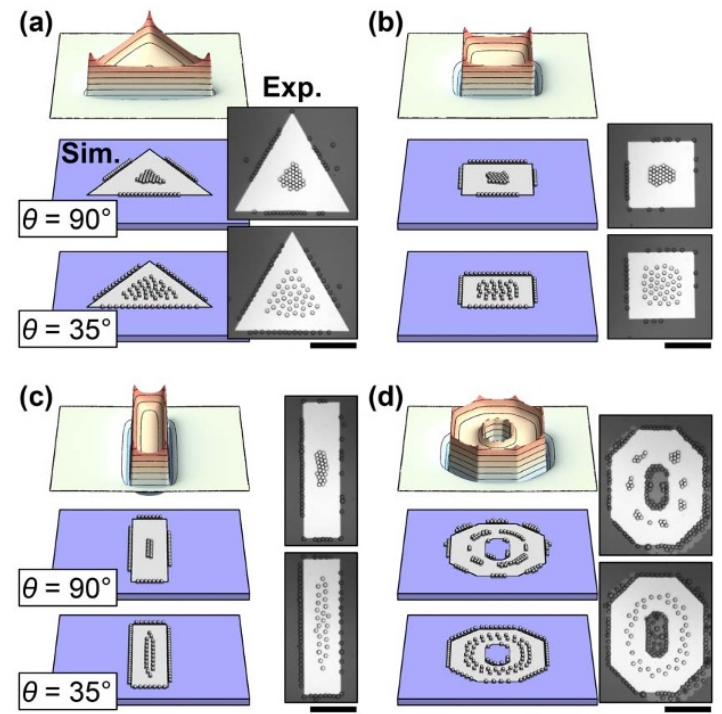

Figure $4 \mid$ Time-orbiting potentials in the shapes of (a) triangle, (b) square, (c) long rectangle, and (d) octagonal ring. Simulations (Sim.) and experimental (Exp.) snapshots of the system in equilibrium are shown below and to the lower-right of the energy landscape in each panel under a precessing $\mathbf{H}_{\text {ext }}$ with $H_{1}=100\left(\times 10^{3} / 4 \pi\right) \mathrm{A} / \mathrm{m}$ and $\theta=90^{\circ}$ or $35^{\circ}$. Contour intervals on the energy landscapes are $2000 k_{\mathrm{B}} T$. Scale bars below the experimental snapshots are $100 \mu \mathrm{m}$. 
previously unexplored opportunities to study field-driven assembly of magnetic dipoles beyond confinements with cylindrical symmetry or simple topology. For instance, the dipole cluster expands to reflect different trap motif and symmetries under repulsive dipolar interaction; the long rectangle serves as a quasi $1 \mathrm{D}$ magnetic channel; the discrete clusters decorating the octagonal ring is a result of the rare competition among inter-particle attractive force and the confinement force - two forces that are typically cooperative. The intricate balance between the interplaying forces and geometry of the softconfining potential leads to the emergence of a complex and designspecific behavior of the dipoles.

The advantages of the all-magnetic approach presented here include easy, non-contact manipulation of the dipoles by weak external magnetic fields, and absence of intricate wiring patterns for addressing specific electrodes as well as screening and heating effects that are generally present in charge-based approaches. We expect the presented scheme to have useful applications, for instances, in biomedical devices ${ }^{31,32}$ : control of local concentration and optimal mixing can be achieved by the expansion and collapse of the dipole cluster; attracting the dipoles from different directions to the center of the disk serves as a simultaneous, multi-directional force probe on a biological entity attached to the center; dipole clusters of tunable spacing can also be fixed as filters to sort out objects of different sizes within a flow channel. From a materials stand point ${ }^{40-42,46,47}$, through multiplexing an array of patterned traps, a two-dimensional field-tunable photonic grating becomes feasible: it offers a length scale relevant at near-visible wavelength and tunable over a factor of two. As its electric counterpart (semiconductor devices) in revolutionizing the electronic age, the all-magnetic scheme presented may also impact the fast-growing fields of magnetic logic devices. The patterned time-orbiting potential could serve as a lithographically defined virtual circuit or wave guide for spins or spin waves in spintronics ${ }^{50}$ and magnonics ${ }^{51}$, while the ability to create clusters of various sizes serves as a strategy to build islands of nanomagnets that are dipole-coupled to produce logic operations $s^{52}$. We further envision the confinement scheme as a potential improvement over conventional traps for magnetic dipolar atoms ${ }^{8-13}$. Given other means to confine the atoms to two dimensions, a $10 \mu \mathrm{m}$ wide circular cavity (inverted disk) on the permalloy film for example, is capable of confining diamagnetic atoms carrying 1 Bohr magneton with an effective spring constant of $10^{-15} \mathrm{~N} / \mathrm{m}$ under $H_{1}=100\left(\times 10^{3} / 4 \pi\right) \mathrm{A} / \mathrm{m}$ and $\theta=45^{\circ}$. Such a pattern-based confinement scheme would allow one to explore the effect of trap geometry on the wave function of the condensate atoms.

In conclusion, we have demonstrated a novel technique based on magnetic thin-film patterns and a precessing field to create timeorbiting potentials of desired architectures with independent control on the confinement and dipolar forces. As advantages over conventional confinement schemes, these lithographically defined softconfining potentials can be remotely activated, micrometer-scaled dipoles loaded or unloaded in-situ and pattern-coded clusters of tunable structures and sizes achieved. With the ability to reversibly and rapidly tune the interplaying forces, structural phenomena are easily reproduced that enable studies in artificial atoms, nucleation, jamming and frustration. We also envision broad applications of this all-magnetic prototype in biomedical devices, assembly of functional materials such as field-tunable photonic gratings, magnetic logic devices, and, when appropriately scaled, trapping of cold quantum gases.

\section{Methods}

Fabrication of permalloy thin-film patterns. Permalloy thin-film patterns are imprinted on a silicon substrate by standard photolithography or electron-beam lithography: For the disk pattern, two layers of photo resist (LOR3B and S1805, MicroChem) are applied to the Si substrate through spin-coating at $2500 \mathrm{rpm}$ followed by baking at $180^{\circ} \mathrm{C}$ and $110^{\circ} \mathrm{C}$ on a hotplate for the respective layers; the disk patterns are then exposed through an optical microscope (BH-2, Olympus) and developed (in MF-319, Dow). For other patterns, two layers of e-beam resist (methylmethacrylate and polymethyl methacrylate, MicroChem) are applied to the $\mathrm{Si}$ substrate through spin coating at $4500 \mathrm{rpm}$ followed by baking on a $180^{\circ} \mathrm{C}$ hotplate; pattern areas are exposed at $125 \mu \mathrm{C} / \mathrm{cm}^{2}$ using a scanning electron microscope (Helios Nanolab 600, FEI Company) and developed using 1:3 methyl isobutyl ketone:isopropyl alcohol (MicroChem). A $40 \mathrm{~nm}$ layer of $\mathrm{Ni}_{0.8} \mathrm{Fe}_{0.2}$ (permalloy) is then deposited onto the resist-patterned surface by magnetron sputtering (ATC Orion, AJA International) followed by lifting off unwanted areas with acetone and $60^{\circ} \mathrm{C}$ bath of $n$-methyl-2-pyrrolidone. The resulting permalloy patterns are protected from the environment by another layer of $\mathrm{SiO}_{2}(100 \sim 200 \mathrm{~nm})$ through spin coating (Silicafilm, Emulsitone) at $4000 \mathrm{rpm}$ and baking at $180^{\circ} \mathrm{C}$ for the disk patterns, or through magnetron sputtering for other patterns. The spin-coated $\mathrm{SiO}_{2}$ surface is treated with $\mathrm{NaOH}(0.1 \mathrm{M})$ for 10 min prior to experiment. The dimensions of various patterns are (in $\mu \mathrm{m})$ : circular disk (210 in diameter), triangle (260 in side length), square $(150 \times 150)$, rectangle $(75 \times 300)$ and octagonal ring $(200 \times 250$ with a $50 \times 100$ hole)

Microsphere solution. Superparamagnetic microspheres (UMC4F/9560, Bangs Laboratories) with diameter of $8 \mu \mathrm{m}$ are diluted in de-ionized water with $0.01 \sim$ $0.05 \%$ Tween-20 (Sigma-Aldrich) for use on spin-coated $\mathrm{SiO}_{2}$ surface or with $0.02 \sim$ $0.05 \%$ Triton X-100 (Sigma-Aldrich) for use on magnetron-sputtered $\mathrm{SiO}_{2}$ surface, preventing surface adhesion. The diluted microsphere solution is then placed on the permalloy-patterned substrate and contained within a polydimethylsiloxane ring capped by a cover glass to eliminate evaporation and fluid flow.

Magnetic field generation. Computer program (LabVIEW, National Instruments) controls the current sent by the power supplies (BOP 20-10ML, Kepco) into the electromagnets and solenoid, providing three-dimensional control of the external magnetic field $\mathrm{H}_{\text {ext }}=\left(H_{x}, H_{y}, H_{z}\right)$ applied on the sample. To prevent net rotation and drifting of the microsphere cluster when a precessing field about the $z$-axis is present, the rotation of the in-plane field is programmed to cycle through the following sequence of angles at a speed of 20 revolutions per second: $180^{\circ},-360^{\circ}, 180^{\circ},-360^{\circ}$, $360^{\circ},-180^{\circ}, 360^{\circ}$ and $-180^{\circ}$. Note that the time period is $0.3 \mathrm{~s}$ per cycle.

1. Brinkman, W., Haggan, D. \& Troutman, W. A history of the invention of the transistor and where it will lead us. IEEE J. Solid-State Circuits 32, 1858-1865 (1997).

2. Mayer, A. M. Floating magnets. Nature 18, 258 (1878)

3. Dimitrov, A. S., Takahashi, T., Furusawa, K. \& Nagayama, K. Two-Dimensional Patterns of Magnetic Particles at Airater or Glassater Interfaces Induced by an External Magnetic Field: Experimental Observation and Dependencies. J. Phys. Chem. 100, 3163-3168 (1996).

4. Bubeck, R., Bechinger, C., Neser, S. \& Leiderer, P. Melting and Reentrant Freezing of Two-Dimensional Colloidal Crystals in Confined Geometry. Phys. Rev. Lett. 82, 3364-3367 (1999).

5. Saado, Y. et al. Self-assembled heterostructures based on magnetic particles for photonic bandgap applications. Opt. Mater. 17, 1-6 (2001).

6. Grzybowski, B. A., Jiang, X., Stone, H. A. \& Whitesides, G. M. Dynamic, selfassembled aggregates of magnetized, millimeter-sized objects rotating at the liquid-air interface: Macroscopic, two-dimensional classical artificial atoms and molecules. Phys. Rev. E: Stat., Nonlinear, Soft Matter Phys. 64, 011603 (2001).

7. Meng, G., Arkus, N., Brenner, M. P. \& Manoharan, V. N. The free-energy landscape of clusters of attractive hard spheres. Science 327, 560-3 (2010).

8. Vassen, W. et al. Cold and trapped metastable noble gases. Rev. Mod. Phys. 84, 175-210 (2012).

9. Minogin, V. G., Richmond, J. A. \& Opat, G. I. Time-orbiting-potential quadrupole magnetic trap for cold atoms. Phys. Rev. A 58, 3138-3145 (1998).

10. Reeves, J. M. et al. Time-orbiting potential trap for Bose-Einstein condensate interferometry. Phys. Rev. A 72, 051605 (2005).

11. Lahaye, T. et al. Strong dipolar effects in a quantum ferrofluid. Nature 448, 672-5 (2007).

12. Koch, T. et al. Stabilization of a purely dipolar quantum gas against collapse. Nat. Phys. 4, 218-222 (2008)

13. Lu, M., Youn, S. H. \& Lev, B. L. Trapping Ultracold Dysprosium: A Highly Magnetic Gas for Dipolar Physics. Phys. Rev. Lett. 104, 063001 (2010).

14. Peeters, F. M., Schweigert, V. A. \& Bedanov, V. M. Classical two-dimensional atoms. Physica B 212, 237-244 (1995).

15. Partoens, B. \& Peeters, F. M. Classical artificial two-dimensional atoms: the Thomson model. J. Phys.: Condens. Matter 9, 5383 (1997).

16. Koulakov, A. A. \& Shklovskii, B. I. Charging spectrum and configurations of a Wigner crystal island. Phys. Rev. B: Condens. Matter Mater. Phys. 57, 2352-2367 (1998).

17. Cremon, J. C., Bruun, G. M. \& Reimann, S. M. Tunable Wigner States with Dipolar Atoms and Molecules. Phys. Rev. Lett. 105, 255301 (2010).

18. Bonitz, M., Henning, C. \& Block, D. Complex plasmas: a laboratory for strong correlations. Rep. Prog. Phys. 73, 66501 (2010).

19. Pusey, P. N. Colloidal Suspensions. Liquids, Freezing and the Glass Transition. Hansen, J.-P., Levesque, D. \& Zinn-Justin, J. (eds.) 763-942 (Elsevier, Amsterdam, 1991).

20. Larsen, A. E. \& Grier, D. G. Like-charge attractions in metastable colloidal crystallites. Nature 385, 230-233 (1997). 
21. Gasser, U., Weeks, E. R., Schofield, A., Pusey, P. N. \& Weitz, D. A. Real-space imaging of nucleation and growth in colloidal crystallization. Science 292, 258-62 (2001)

22. Zhang, K.-Q. \& Liu, X. Y. In situ observation of colloidal monolayer nucleation driven by an alternating electric field. Nature 429, 739-43 (2004).

23. Reichhardt, C. J. O., Reichhardt, C. \& Bishop, A. R. Structure and fragmentation in colloidal artificial molecules and nuclei. Eur. Phys. J. E: Soft Matter Biol. Phys. 22, 11-15 (2007).

24. Wang, Z., Wang, F., Peng, Y., Zheng, Z. \& Han, Y. Imaging the homogeneous nucleation during the melting of superheated colloidal crystals. Science 338, 87-90 (2012).

25. Liu, A. J. \& Nagel, S. R. Nonlinear dynamics: Jamming is not just cool any more. Nature 396, 21-22 (1998).

26. Weitz, D. A. \& Oliveria, M. Fractal Structures Formed by Kinetic Aggregation of Aqueous Gold Colloids. Phys. Rev. Lett. 52, 1433-1436 (1984).

27. Segré, P. N., Prasad, V., Schofield, A. B. \& Weitz, D. A. Glasslike Kinetic Arrest at the Colloidal-Gelation Transition. Phys. Rev. Lett. 86, 6042-6045 (2001).

28. Lubchenko, V. Competing interactions create functionality through frustration. Proc. Natl. Acad. Sci. U. S. A. 105, 10635-6 (2008).

29. Muthukumar, M. Competing Interactions and Levels of Ordering in SelfOrganizing Polymeric Materials. Science 277, 1225-1232 (1997).

30. Toledano, J. C. F., Sciortino, F. \& Zaccarelli, E. Colloidal systems with competing interactions: from an arrested repulsive cluster phase to a gel. Soft Matter 5, 2390 (2009).

31. Gijs, M. A. M. Magnetic bead handling on-chip: new opportunities for analytical applications. Microfluid. Nanofluid. 1, 22-40 (2004).

32. Pamme, N. Magnetism and microfluidics. Lab Chip 6, 24-38 (2006).

33. Ramadan, Q. \& Gijs, M. A. M. Simultaneous sample washing and concentration using a "trapping-and-releasing" mechanism of magnetic beads on a microfluidic chip. Analyst (Cambridge, U. K.) 136, 1157-66 (2011).

34. Vieira, G. et al. Magnetic wire traps and programmable manipulation of biological cells. Phys. Rev. Lett. 103, 128101 (2009).

35. Hoi, S.-K. et al. A Microfluidic Chip with Integrated Colloidal Crystal for Online Optical Analysis. Adv. Funct. Mater. 21, 2847-2853 (2011).

36. Kinnunen, P. et al. Self-assembled magnetic bead biosensor for measuring bacterial growth and antimicrobial susceptibility testing. Small 8, 2477-82 (2012).

37. Seo, Y. H. Nanomesh fluidic filter using self-assembly of colloidal nanospheres and surface tension. Appl. Phys. Lett. 90, 123514 (2007).

38 . He, J. et al. Diffusion and filtration properties of self-assembled gold nanocrystal membranes. Nano Lett. 11, 2430-5 (2011).

39. Issadore, D. et al. Self-assembled magnetic filter for highly efficient immunomagnetic separation. Lab Chip 11, 147-51 (2011).

40. Whitesides, G. M. \& Boncheva, M. Beyond molecules: self-assembly of mesoscopic and macroscopic components. Proc. Natl. Acad. Sci. U. S. A. 99 4769-74 (2002).

41. Glotzer, S. C. Some Assembly Required. Science 306, 419-20 (2004).

42. Li, F., Josephson, D. P. \& Stein, A. Colloidal assembly: the road from particles to colloidal molecules and crystals. Angew. Chem., Int. Ed. 50, 360-88 (2011).

43. Martin, J. E., Anderson, R. A. \& Williamson, R. L. Generating strange magnetic and dielectric interactions: Classical molecules and particle foams. J. Chem. Phys. 118, 1557 (2003).

44. Osterman, N. et al. Field-Induced Self-Assembly of Suspended Colloidal Membranes. Phys. Rev. Lett. 103, 228301 (2009).

45. Khalil, K. S. et al. Binary colloidal structures assembled through Ising interactions. Nat. Commun. 3, 794 (2012).

46. Joannopoulos, J. D., Meade, R. D. \& Winn, J. N. Photonic Crystals: Molding the Flow of Light (Princeton University Press, 1995).

47. Ge, J. \& Yin, Y. Responsive photonic crystals. Angew. Chem., Int. Ed. 50, 1492-522 (2011).

48. Arsenault, A. C., Puzzo, D. P., Manners, I. \& Ozin, G. A. Photonic-crystal fullcolour displays. Nat. Photonics 1, 468-472 (2007).
49. Weissman, J. M., Sunkara, H. B., Tse, A. S. \& Asher, S. A. Thermally Switchable Periodicities and Diffraction from Mesoscopically Ordered Materials. Science 274, 959-963 (1996).

50. Flatté, M. E. Solid-state physics: Silicon spintronics warms up. Nature 462, 419-20 (2009).

51. Kruglyak, V. V., Demokritov, S. O. \& Grundler, D. Magnonics. J. Phys. D: Appl. Phys. 43, 264001 (2010)

52. Carlton, D. et al. Signal propagation in dipole coupled nanomagnets for logic applications. Proc. SPIE 8461, 84610W (2012)

53. Rapoport, E., Montana, D. \& Beach, G. S. D. Integrated capture, transport, and magnetomechanical resonant sensing of superparamagnetic microbeads using magnetic domain walls. Lab on a chip 12, 4433-40 (2012).

54. Vavassori, P. et al. Domain wall displacement in Py square ring for single nanometric magnetic bead detection. Applied Physics Letters 93, 203502 (2008)

55. Donolato, M. et al. On-chip manipulation of protein-coated magnetic beads via domain-wall conduits. Advanced materials 22, 2706-10 (2010).

56. Donolato, M. et al. Magnetic domain wall conduits for single cell applications. Lab on a chip 11, 2976-83 (2011).

57. Chen, A. et al. On-chip magnetic separation and encapsulation of cells in droplets. Lab on a chip 13, 1172-81 (2013).

58. Joseph, R. I. \& Schlömann, E. Demagnetizing field in nonellipsoidal bodies. Journal of Applied Physics 36, 1579-1593 (1965).

59. Guslienko, K. Y. \& Slavin, A. N. Spin-waves in cylindrical magnetic dot arrays with in-plane magnetization. Journal of Applied Physics 87, 6337 (2000).

60. Zivieri, R. \& Stamps, R. Theory of spin wave modes in tangentially magnetized thin cylindrical dots: A variational approach. Physical Review B 73, 144422 (2006).

61. Delaunay, B. Sur la sphére vide. Izv. Akad. Nauk SSSR, Otd. Mat. Estestv. Nauk 7, 793-800 (1934).

62. Thomson, J. J. On the structure of the atom: an investigation of the stability and periods of oscillation of a number of corpuscles arranged at equal intervals around the circumference of a circle; with application of the results to the theory of atomic structure. Philos. Mag. (1798-1977) 7, 237-265 (1904).

\section{Acknowledgments}

This material is based upon work supported by the U.S. Army Research Office under contract W911NF-10-1-0353 and the NSF NSEC grant number EEC-0914790. We appreciate T. Henighan and G. Vieira for the assistances in experiment and fabrication, and Y.-H. Lin for the useful discussions.

\section{Author contributions}

R.S. initiated and supervised the project. A.C. conducted the experiments, analyzed the data and developed the model. A.C. and R.S. wrote the paper.

\section{Additional information}

Supplementary information accompanies this paper at http://www.nature.com/ scientificreports

Competing financial interests: The authors declare no competing financial interests.

How to cite this article: Chen, A. \& Sooryakumar, R. Patterned time-orbiting potentials for the confinement and assembly of magnetic dipoles. Sci. Rep. 3, 3124; DOI:10.1038/ srep03124 (2013).

(C) $(9)$ This work is licensed under a Creative Commons Attribution-

BY NC ND NonCommercial-NoDerivs 3.0 Unported license. To view a copy of this license, visit http://creativecommons.org/licenses/by-nc-nd/3.0 